\title{
Acute pulmonary thromboemboli due to a giant mobile thrombus
}

\section{Ataman Köse', Mehmet Kerem Karaca ${ }^{2}$, Ahmet Celik ${ }^{3}$, Serdar Biricik', Murat Ozeren ${ }^{2}$}

'Department of Emergency Medicine, Mersin University School of Medicine, Mersin, Turkey ${ }^{2}$ Department of Cardiovascular Surgery, Mersin University School of Medicine, Mersin, Turkey ${ }^{3}$ Department of Cardiology, Mersin University School of Medicine, Mersin, Turkey

A 51-year-old man presented to the emergency department with progressive dyspnea and orthopnea that had persisted for 15 days. The patient had no history of any disease. Upon physical examination, he had a brachial blood pressure of $80 / 50 \mathrm{mmHg}$, a heart rate of 130/min, and a respiratory rate of 40/min; a 2/6 pansystolic murmur was heard in the mesocardiac region, and breath sounds were decreased in the patient's lungs. An electrocardiography revealed sinus tachycardia and a right bundle branch block. The patient's arterial blood gas measurements were as follows: $\mathrm{pH}_{1} 7.52 ; \mathrm{pO}_{2}, 61.8 \mathrm{mmHg} ; \mathrm{pCO}_{2}, 20.5 \mathrm{mmHg} ; \mathrm{HCO}_{3}^{-}, 16.6 \mathrm{mmHg}$; and $\mathrm{O}_{2}$ saturation, $93 \%$. With regards to other blood parameters, biochemical analysis revealed a hemoglobin level of $11.4 \mathrm{~g} / \mathrm{dL}$, a white blood cell count of $12,990 / \mu \mathrm{L}$, a platelet count of $169,000 / \mu \mathrm{L}$, a serum creatinine level of $0.97 \mathrm{mg} / \mathrm{dL}$, a creatinine kinase-MB level of $2.78 \mathrm{ng} / \mathrm{mL}$ (within the normal range), a troponin I level of $0.449 \mathrm{ng} / \mathrm{mL}$, and a D-dimer level of $>5,000 \mathrm{ng} / \mathrm{mL}$. We carried out echocardiography, which revealed a 60\% left ventricular ejection fraction, a moderated tricuspid regurgitation, and a systolic pulmonary artery pressure of $70 \mathrm{mmHg}$, with an enlarged right ventricle. The interventricular septum was semi-paradoxical, and the right ventricular free wall was hypokinetic. A $7 \times 1.5 \mathrm{~cm}$, free-floating giant thrombus was seen extending from the inferior vena cava to the right atrium and right ventricle (Fig. 1). A mobile thrombus was also seen in the
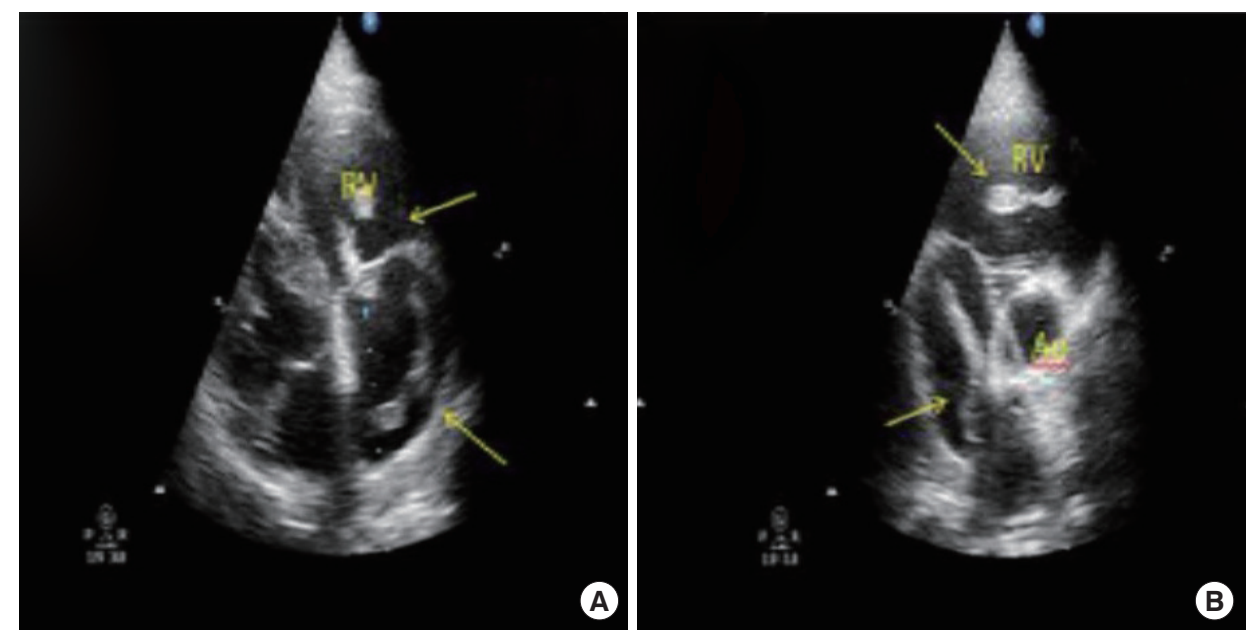

Fig. 1. A $7 \times 1.5 \mathrm{~cm}$, free-floating giant thrombus is seen extending from the inferior vena cava to the RA and RV. (A) Four chamber view, arrow: thrombus in RA and RV. (B) Parasternal short axis view, arrow: thrombus in $\mathrm{RV}$ and pulmonary artery. $\mathrm{RA}$, right atrium; $\mathrm{RV}$, right ventricle.
Received: 5 December 2016 Revised: 7 February 2017

Accepted: 14 February 2017

Correspondence to: Ataman Köse Department of Emergency Medicine, Mersin University School of Medicine, Mersin, Turkey

E-mail: ataberk76@yahoo.com.tr

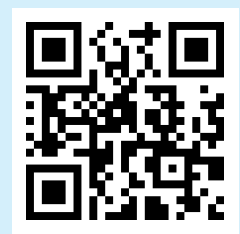

How to cite this article:

Köse A, Karaca MK, Celik A, Biricik S, Ozeren M. Acute pulmonary thromboemboli due to a giant mobile thrombus. Clin Exp Emerg Med 2017;4(2):117-118.

This is an Open Access article distributed under the terms of the Creative Commons Attribution Non-Commercial License (http:// creativecommons.org/licenses/by-nc/4.0/). 


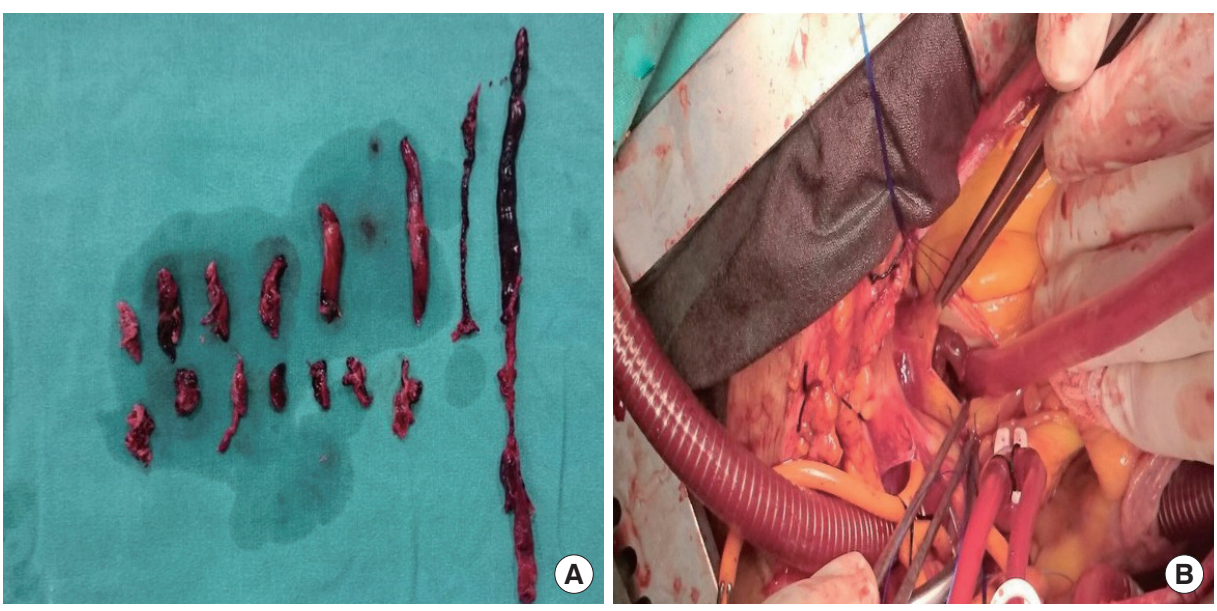

Fig. 2. The patient underwent emergency surgical thrombectomy, and the thrombus was removed. (A) Surgical thrombectomy specimen and (B) exploration of right atrium during surgery.

proximal right pulmonary artery. We diagnosed acute, high-risk pulmonary embolism. The patient underwent emergency surgical thrombectomy, and the thrombus was removed (Fig. 2).

Pulmonary embolism (PE) is a common, potentially fatal condition. Early diagnosis and anticoagulation therapy can reduce mortality from $30 \%$ to $2 \%$ to $10 \%{ }^{1,2}$ However, in emergency rooms, it is not always possible to access the definitive diagnostic tools for acute pulmonary embolism. Arterial blood gas analysis, electrocardiogram, and chest radiography are not reliable tools for detecting PE. Moreover, D-dimer testing is less useful in patients who are already hospitalized, because D-dimer levels are elevated in many illnesses that mimic PE. ${ }^{3}$ Scintigraphy and multidetector computed tomography might be difficult and costly to perform and interpret in emergency departments in some countries. In addition, they are not suitable for every patient (particularly for unstable patients); contrast agents is another problem with these techniques.

Transthoracic echocardiography is a useful method for diagnosing PE in emergency rooms. It can be used to evaluate right ventricular morphology and function, as well as paradoxical movement of the interventricular septum. It is a rapid, practical, and sensitive technique for quickly identifying right heart thrombi. Echocardiography may also help to exclude other diagnoses, such as aortic dissection, pericardial disease, myocardial infarction, and valvular insufficiency. ${ }^{4}$ In unstable patients, emergency department doctors can perform the technique quickly at the bedside.

Giant, mobile, thrombus-related pulmonary embolisms are rare. If the giant thrombus is in the right atrium, mortality can be as high as $45 \% 0^{5,6}$ Indeed, the mortality rate is $80 \%$ to $100 \%$ in untreated patients with mobile thrombus in the right side of the heart. The treatment options for these patients include fibrinolytic therapy, surgery, and anticoagulant therapy. If an emergency physician diagnoses PE quickly using ultrasound, he/she will be able to treat the patient quickly, especially if surgery is necessary.

\section{CONFLICT OF INTEREST}

No potential conflict of interest relevant to this article was reported.

\section{REFERENCES}

1. Fedullo PF, Tapson VF. Clinical practice: the evaluation of suspected pulmonary embolism. N Engl J Med 2003;349:124756.

2. Kavanagh EC, O'Hare A, Hargaden G, Murray JG. Risk of pulmonary embolism after negative MDCT pulmonary angiography findings. AJR Am J Roentgenol 2004;182:499-504.

3. Sohns C, Amarteifio E, Sossalla $S$, Heuser M, Obenauer S. 64-Multidetector-row spiral CT in pulmonary embolism with emphasis on incidental findings. Clin Imaging 2008;32:335-41.

4. Mansencal N, Joseph T, Vieillard-Baron A, et al. Comparison of different echocardiographic indexes secondary to right ventricular obstruction in acute pulmonary embolism. Am J Cardiol 2003;92:116-9.

5. Barriales V, Tamargo JA, Aguado MG, et al. Floating thrombi on the Eustachian valve as a complication of venous thromboembolic disease. Int J Cardiol 2004;93:289-91.

6. Chartier L, Bera J, Delomez M, et al. Free-floating thrombi in the right heart: diagnosis, management, and prognostic indexes in 38 consecutive patients. Circulation 1999;99:2779-83.

7. Rose PS, Punjabi NM, Pearse DB. Treatment of right heart thromboemboli. Chest 2002;121:806-14. 